\title{
Article \\ Germline PALB2 Mutation in High-Risk Chinese Breast and/or Ovarian Cancer Patients
}

\author{
Ava Kwong $1,2,3,4, *$, Vivian Y. Shin ${ }^{1,2}$, Cecilia Y. S. Ho ${ }^{5}$, Aleena Khalid ${ }^{1,2}{ }^{\circledR}$, Chun Hang Au ${ }^{5}$, Karen K. L. Chan ${ }^{6}$, \\ Hextan Y. S. Ngan ${ }^{6}$, Tsun-Leung Chan ${ }^{4,5}$ and Edmond S. K. Ma ${ }^{4,5}$
}

1 Department of Surgery, The University of Hong Kong, Hong Kong, China; vyshin@hku.hk (V.Y.S.); u3549370@connect.hku.hk (A.K.)

2 University of Hong Kong-Shenzhen Hospital, Hong Kong, China

3 Department of Surgery, Hong Kong Sanatorium \& Hospital, Hong Kong, China

4 Hong Kong Hereditary Breast Cancer Family Registry, Hong Kong, China;

Chris.TL.Chan@hksh.com (T.-L.C.); eskma@hksh.com (E.S.K.M.)

5 Department of Pathology, Division of Molecular Pathology, Hong Kong Sanatorium \& Hospital, Hong Kong, China; cecilia.ys.ho@hksh.com (C.Y.S.H.); Tommy.CH.Au@hksh.com (C.H.A.)

6 Department of Obstetrics and Gynecology, The University of Hong Kong, Hong Kong, China; kklchan@hku.hk (K.K.L.C.); hysngan@hku.hk (H.Y.S.N.)

* Correspondence: akwong@asiabreastregistry.com; Tel.: +852-2255-4773

check for

updates

Citation: Kwong, A.; Shin, V.Y.; Ho, C.Y.S.; Khalid, A.; Au, C.H.; Chan, K.K.L.; Ngan, H.Y.S.; Chan, T.-L.; Ma, E.S.K. Germline PALB2 Mutation in High-Risk Chinese Breast and/or Ovarian Cancer Patients. Cancers 2021, 13, 4195. https://doi.org/ 10.3390/cancers13164195

Academic Editor:

Dionyssios Katsaròs

Received: 16 July 2021

Accepted: 17 August 2021

Published: 20 August 2021

Publisher's Note: MDPI stays neutral with regard to jurisdictional claims in published maps and institutional affiliations.

Copyright: (c) 2021 by the authors. Licensee MDPI, Basel, Switzerland. This article is an open access article distributed under the terms and conditions of the Creative Commons Attribution (CC BY) license (https:// creativecommons.org/licenses/by/ $4.0 /)$.
Simple Summary: Breast cancer is the most commonly diagnosed cancer in women globally. BRCA1 and $B R C A 2$ mutations are most prevalent in hereditary breast cancer and are associated with increased risk of breast and ovarian cancer. The PALB2 (partner and localizer of BRCA2) mutation was found to be associated with an increased risk of breast cancer and one of the most common mutations, after $B R C A 1$ and $B R C A 2$, in non-BRCA1/2 breast cancer patients. The prevalence of the PALB2 mutation in breast cancer varies across different ethnic groups; hence, it is of intense interest to evaluate the cancer risk and clinical association of PALB2 mutation in Chinese breast and/or ovarian cancer patients.

Abstract: The prevalence of the PALB2 mutation in breast cancer varies across different ethnic groups; hence, it is of intense interest to evaluate the cancer risk and clinical association of the PALB2 mutation in Chinese breast and/or ovarian cancer patients. We performed sequencing with a 6-gene test panel (BRCA1, BRCA2, TP53, PTEN, PALB2, and CDH1) to identify the prevalence of the PALB2 germline mutation among 2631 patients with breast and/or ovarian cancer. In this cohort, 39 mutations were identified with 24 types of mutation variants, where the majority of the mutations were frame-shift mutations and resulted in early termination. We also identified seven novel PALB2 mutations. Most of the PALB2 mutation carriers had breast cancer $(36,92.3 \%)$ and were more likely to have family history of breast cancer $(19,48.7 \%)$. The majority of the breast tumors were invasive ductal carcinoma (NOS type) (34, 81.0\%) and hormonal positive (ER: 32, 84.2\%; PR: 23, 60.5\%). Pathogenic mutations of PALB2 were found in 39 probands with a mutation frequency of $1.6 \%$ and $1 \%$ in breast cancer and ovarian cancer patients, respectively. PALB2 mutation carriers were more likely have hormonal positive tumors and were likely to have familial aggregation of breast cancer.

Keywords: hereditary breast cancer; PALB2 mutation; Chinese; breast cancer risk

\section{Introduction}

Breast cancer is a growing public health concern, as it is the most commonly diagnosed cancer in women globally. Inherited gene mutation is one of the risk factors for developing breast cancer or ovarian cancer in women. Over the past decades, a number of mutations have been identified in hereditary breast cancer, among which $B R C A 1$ and $B R C A 2$ mutations are the most prevalent and associated with increased risk of breast and ovarian cancer [1]. Genetic testing is becoming more important, since the test results greatly impact the clinical management of high-risk patients and their family members. With 
the emergence of next-generation sequencing (NGS), utilization of multigene panel with high-penetrance genes (e.g., $B R C A$ ) and moderate- or low-penetrance genes are included for cancer diagnosis. The PALB2 (partner and localizer of BRCA2) mutation was found to be associated with an increased risk of breast cancer and one of the most common mutations, after $B R C A 1$ and $B R C A 2$, in non-BRCA1/2 breast cancer patients [2-5]. The clinical evidence of $P A L B 2$ variants is increasing, and the clinical guidelines and management for PALB2 mutation carriers are mainly based on consensus but still lack solid supportive evidence. Hence, additional studies that allow the mutation spectrum and the clinical outcomes of PALB2 mutation carriers to be understood has implication for public health.

The PALB2 gene encodes for a tumor suppressor protein that binds to and co-localizes with BRCA2 in the nucleus and assists in the formation of the BRCA1-PALB2-BRCA2 complex [6]. It is Fanconi anemia (FA) gene located on 16q12.22, which plays a key role in double strand break repair by homologous recombination alongside BRCA1/2 [7]; hence, mutations in PALB2 lead to defects in DNA repair pathways. Biallelic mutations in PALB2 gene lead to FA subtype-D1 (FA-D1) and childhood solid tumors, whereas the monoallelic mutation has been linked to hereditary breast and ovarian cancer syndrome [8,9]. The lifetime risk of developing breast cancer in women with the PALB2 mutation is $52.8 \%$ by age of 80 [3].

The prevalence and frequency of the PALB2 mutation in breast cancer varies across different ethnic groups [10]. According to a study performed in the Finnish population, PALB2 c.159delT was identified as the founder mutation in the respective population [11]. Similarly, a PALB2 screening study performed in 1403 female Australian breast cancer patients followed by screening of 779 families revealed that PALB2 c.3113G $>$ A was the most recurrent familial mutation [12]. Moreover, PALB2 mutations accounted for $1.1 \%$ of the breast cancer cases in the Caucasian population [13]. Our recent study reported that the mutation frequency of PALB2 was $1.4 \%$, which was the most frequently mutated gene after $B R C A 1$ and BRCA2 genes in Chinese patients [14]. Similarly, studies showed that PALB2 mutations account for approximately $1-2 \%$ of early onset breast cancer patients in the Chinese population $[10,15]$. Thus, understanding the cancer risk and phenotypic presentations of the PALB2 mutation in Chinese breast cancer contribute to clinical management decisions.

To date, no effective therapeutic strategies have been devised for PALB2 mutationcarrying breast cancer patients, despite a few case studies suggesting the use of platinumbased chemotherapies in the metastatic setting. Recently, there was a report of a PALB2 mutation-carrying breast cancer patient treated with carboplatin for 7 months, whom after relapse showed complete response and disease-free post 30-months of treatment [16]. Similarly, another patient with invasive ductal carcinoma, post relapse, upon treatment with carboplatin showed complete response. Unfortunately, due to recurrent thrombocytopenia, the treatment was stopped, and the patient passed away. Another study showed promising results with the use of PARP inhibitor (olaparib) in a breast cancer patient with the PALB2 mutation [17]. These studies suggest that platinum-based therapies and PARP inhibitors could potentially be used to treat PALB2 mutation carriers. Better understanding of PALB2 mutation carriers would allow for better evidence to support clinical guidelines for the management of such carriers.

\section{Results}

\subsection{Patients' Characteristics of the Cohort}

Our testing cohort included 2631 patients with breast cancer and/or ovarian cancer. The median age at diagnosis of breast cancer was 43 years (range 18-88) and 49 years (range 9-85) for ovarian cancer. Of these, 2105 (80.0\%) were breast cancer patients, $398(15.1 \%)$ were ovarian cancer patients, and $128(4.9 \%)$ were diagnosed with both breast and ovarian cancers. Bilateral breast cancers were seen in 552 patients $(24.7 \%)$. The majority of the breast cancers were ductal carcinoma (NOS Type) $(1872,69.7 \%)$. There was a high percentage of breast cancers, which were found to be hormonal positive $(1525,70.8 \%)$ followed by triple-negative breast cancers (TNBC) (430, 20.0\%). Most of the breast tumors 
were diagnosed at early stages ( 0 , I, or II) $(2203,85.0 \%)$ and grading favor grade 2 or 3 $(858,45.6 \%$ and $722,38.4 \%$, respectively). Most of the ovarian cancers were diagnosed with epithelial cancers $(471,96.3 \%)$ and the majority of high grade $(294,64.2 \%)$. A positive family history of breast cancer (first- or second-degree relatives) was seen among 952 patients (36.2\%). Family history of ovarian cancer and prostate cancer were also seen in $162(6.2 \%)$ and $123(4.7 \%)$, respectively, of their relatives. Detailed clinicopathological characteristics are shown in Table 1.

Table 1. Clinicopathologic characteristics of study cohort.

\begin{tabular}{|c|c|c|}
\hline Clinicopathologic Characteristics & $n=2631$ & $\%$ \\
\hline First diagnosis of breast cancer & $45.1 / 43$ & \\
\hline Mean/Median (Range) & $(18-88)$ & \\
\hline First diagnosis of ovarian cancer & $48.5 / 49$ & \\
\hline Mean/Median (Range) & $(9-85)$ & \\
\hline \multicolumn{3}{|c|}{ Gender } \\
\hline Female & 2564 & $97.5 \%$ \\
\hline Male & 67 & $2.5 \%$ \\
\hline \multicolumn{3}{|c|}{ Personal Cancer } \\
\hline Breast Cancer & 2105 & $80.0 \%$ \\
\hline Ovarian Cancers & 398 & $15.1 \%$ \\
\hline Breast and Ovarian Cancers & 128 & $4.9 \%$ \\
\hline Bilateral CA & 552 & $24.7 \%$ \\
\hline \multicolumn{3}{|c|}{ Family history of cancers in 1st and 2nd degree } \\
\hline Breast Cancer & 952 & $36.2 \%$ \\
\hline Ovarian Cancer & 162 & $6.2 \%$ \\
\hline Prostate Cancer & 123 & $4.7 \%$ \\
\hline Liver Cancer & 288 & $10.9 \%$ \\
\hline Stomach Cancer & 224 & $8.5 \%$ \\
\hline Lung Cancer & 528 & $20.1 \%$ \\
\hline Colorectal Cancer & 463 & $17.6 \%$ \\
\hline \multicolumn{3}{|c|}{ Pathology of Breast Cancers $(n=2785) *$} \\
\hline \multicolumn{3}{|c|}{ Breast cancer histology } \\
\hline Ductal & 1872 & $69.7 \%$ \\
\hline In situ & 465 & $17.3 \%$ \\
\hline Others & 349 & $13.0 \%$ \\
\hline \multicolumn{3}{|c|}{ Grade } \\
\hline 1 & 301 & $16.0 \%$ \\
\hline 2 & 858 & $45.6 \%$ \\
\hline 3 & 722 & $38.4 \%$ \\
\hline \multicolumn{3}{|c|}{ Molecular Subtype } \\
\hline TNBC & 430 & $20.0 \%$ \\
\hline HER2+ & 162 & $7.5 \%$ \\
\hline ER/PR+ & 1525 & $70.8 \%$ \\
\hline \multicolumn{3}{|c|}{ Stage } \\
\hline 0 & 483 & $18.6 \%$ \\
\hline 1 & 954 & $36.8 \%$ \\
\hline 2 & 766 & $29.6 \%$ \\
\hline 3 & 289 & $11.1 \%$ \\
\hline 4 & 100 & $3.9 \%$ \\
\hline
\end{tabular}


Table 1. Cont.

\begin{tabular}{ccc}
\hline $\begin{array}{c}\text { Clinicopathologic } \\
\text { Characteristics }\end{array}$ & $n=2631$ & $\%$ \\
\hline & Pathology of Ovarian Cancers $(n=526)$ \\
\hline & Ovarian cancer histology \\
Epithelial & 471 & $96.3 \%$ \\
Germ Cell & 7 & $1.4 \%$ \\
Stromal & 5 & $1.0 \%$ \\
Mixed & 6 & $1.2 \%$ \\
Not stated & 37 & - \\
& Grade & \\
1 & 51 & $11.1 \%$ \\
2 & 107 & $23.4 \%$ \\
3 & 294 & $64.2 \%$ \\
Mixed & 6 & $1.3 \%$ \\
Not stated & 68 & - \\
& Stage & \\
1 & 201 & $42.4 \%$ \\
2 & 59 & $12.4 \%$ \\
3 & 157 & $33.1 \%$ \\
4 & 57 & $12.0 \%$ \\
Not stated & 52 & - \\
\hline *552 bilateral breast cancers. TNBC: triple-negative breast cancer; HER2: human epidermal growth factor receptor
\end{tabular}
2; ER: estrogen receptors; PR: progesterone receptors.

\subsection{Characteristics of Mutation Carriers}

Heterozygous pathogenic mutations of PALB2 were found in 39 probands with a mutation frequency of $1.6 \%$ among high-risk breast and $1 \%$ of ovarian cancer patients, and there was one patient with double heterozygous mutations identified in BRCA1 and PALB2. Among the single mutation carriers, the median age of breast cancer diagnosis was 39 (range 24-69) and 59 (range 36-65) for ovarian cancer mutation carriers; all of the PALB2 carriers were female (Table 2 ). The majority of the cases are patients with breast cancer $(36,92.3 \%)$ and 5 out of 39 (12.8\%) with ovarian cancer. Four of the PALB2 carriers had multiple cancers including breast cancer, ovarian cancer, colorectal cancer, or cancer in the uterus. The percentage of bilateral breast cancers in the carriers $(27.8 \%)$ was similar to the non-carriers $(23.5 \%)$. A positive family history of breast cancer (first- or second-degree relatives) was seen among 19 of the patients (48.7\%). None of them had a family history of ovarian cancers in their relatives.

Table 2. Characteristics and mutation frequencies of PALB2 and BRCA1/2.

\begin{tabular}{|c|c|c|c|c|c|c|c|c|c|c|c|c|c|c|}
\hline \multirow[t]{3}{*}{ No. of Probands } & \multirow{2}{*}{\multicolumn{2}{|c|}{$\begin{array}{c}n=39 \\
\text { PALB2+ }\end{array}$}} & \multirow{2}{*}{\multicolumn{2}{|c|}{$\begin{array}{c}n=173 \\
\text { BRCA1+ }\end{array}$}} & \multirow{2}{*}{\multicolumn{2}{|c|}{$\begin{array}{l}n=204 \\
B R C A 2+\end{array}$}} & \multirow{2}{*}{\multicolumn{2}{|c|}{$\begin{array}{l}n=377 \\
B R C A+\end{array}$}} & \multirow{2}{*}{\multicolumn{2}{|c|}{$\begin{array}{c}n=2214 \\
\text { Negative }\end{array}$}} & \multicolumn{4}{|c|}{$p$-Value } \\
\hline & & & & & & & & & & & \multirow{2}{*}{$\begin{array}{c}\text { PALB2+ vs. } \\
\text { BRCA1+ }\end{array}$} & \multirow{2}{*}{$\begin{array}{c}\text { PALB2+ vs. } \\
\text { BRCA2+ }\end{array}$} & \multirow{2}{*}{$\begin{array}{c}\text { PALB2+ vs. } \\
\text { BRCA+ }\end{array}$} & \multirow{2}{*}{$\begin{array}{l}\text { PALB2+ vs. } \\
\text { Negative }\end{array}$} \\
\hline & $\mathrm{n}$ & $\%$ & $\mathrm{n}$ & $\%$ & $\mathbf{n}$ & $\%$ & $\mathrm{n}$ & $\%$ & $\mathbf{n}$ & $\%$ & & & & \\
\hline \multicolumn{15}{|c|}{ Breast cancer first DX } \\
\hline $\begin{array}{l}\text { Mean/Median } \\
\text { (Range) }\end{array}$ & $\begin{array}{l}43.5 / 39 \\
(24-69)\end{array}$ & & $\begin{array}{l}41.0 / 40 \\
(22-73)\end{array}$ & & $\begin{array}{l}43.0 / 42 \\
(21-73)\end{array}$ & & $\begin{array}{l}42.2 / 41 \\
(21-73)\end{array}$ & & $\begin{array}{c}45.7 / 43 \\
(8-88)\end{array}$ & & 0.466 & 0.616 & 0.984 & 0.227 \\
\hline \multicolumn{15}{|c|}{ Ovarian cancer first DX } \\
\hline $\begin{array}{l}\text { Mean/Median } \\
\text { (Range) }\end{array}$ & $\begin{array}{l}53.6 / 59 \\
(36-65)\end{array}$ & & $\begin{array}{l}51.4 / 51 \\
(17-85)\end{array}$ & & $\begin{array}{l}53.4 / 53 \\
(31-74)\end{array}$ & & $\begin{array}{l}52.1 / 51 \\
(17-85)\end{array}$ & & $\begin{array}{c}47.3 / 48 \\
(9-79)\end{array}$ & & NA* & $\mathrm{NA}^{*}$ & $\mathrm{NA}^{*}$ & $\mathrm{NA}^{*}$ \\
\hline \multicolumn{15}{|c|}{ Gender } \\
\hline Female & 39 & $100.0 \%$ & 173 & $100.0 \%$ & 190 & $93.1 \%$ & 363 & $96.3 \%$ & 2161 & $97.6 \%$ & \multirow{2}{*}{1.000} & \multirow{2}{*}{0.135} & \multirow{2}{*}{0.381} & \multirow{2}{*}{1.000} \\
\hline Male & 0 & $0.0 \%$ & 0 & $0.0 \%$ & 14 & $6.9 \%$ & 14 & $3.7 \%$ & 53 & $2.4 \%$ & & & & \\
\hline \multicolumn{15}{|c|}{ Personal cancer of breast or ovarian cancer } \\
\hline Breast & 34 & $87.2 \%$ & 93 & $53.8 \%$ & 163 & $79.9 \%$ & 256 & $67.9 \%$ & 1815 & $82.0 \%$ & \multirow{3}{*}{$<0.001$} & \multirow{3}{*}{0.749} & \multirow{3}{*}{0.050} & \multirow{3}{*}{0.356} \\
\hline $\begin{array}{l}\text { Breast and } \\
\text { Ovarian }\end{array}$ & 2 & $5.1 \%$ & 34 & $19.7 \%$ & 16 & $7.8 \%$ & 50 & $13.3 \%$ & 75 & $3.4 \%$ & & & & \\
\hline Ovarian & 3 & $7.7 \%$ & 46 & $26.6 \%$ & 25 & $12.3 \%$ & 71 & $18.8 \%$ & 324 & $14.6 \%$ & & & & \\
\hline
\end{tabular}


Table 2. Cont.

\begin{tabular}{|c|c|c|c|c|c|c|c|c|c|c|c|c|c|c|}
\hline \multirow[t]{3}{*}{ No. of Probands } & \multirow{2}{*}{\multicolumn{2}{|c|}{$\begin{array}{c}n=39 \\
\text { PALB2+ }\end{array}$}} & \multirow{2}{*}{\multicolumn{2}{|c|}{$\begin{array}{c}n=173 \\
\text { BRCA1+ }\end{array}$}} & \multirow{2}{*}{\multicolumn{2}{|c|}{$\begin{array}{l}n=204 \\
B R C A 2+\end{array}$}} & \multirow{2}{*}{\multicolumn{2}{|c|}{$\begin{array}{l}n=377 \\
B R C A+\end{array}$}} & \multirow{2}{*}{\multicolumn{2}{|c|}{$\begin{array}{c}n=2214 \\
\text { Negative }\end{array}$}} & \multicolumn{4}{|c|}{$p$-Value } \\
\hline & & & & & & & & & & & \multirow{2}{*}{$\begin{array}{c}\text { PALB2+ vs. } \\
\text { BRCA1+ }\end{array}$} & \multirow{2}{*}{$\begin{array}{c}\text { PALB2+ vs. } \\
\text { BRCA2+ }\end{array}$} & \multirow{2}{*}{$\begin{array}{c}\text { PALB2+ vs. } \\
\text { BRCA+ }\end{array}$} & \multirow{2}{*}{$\begin{array}{c}P A L B 2+\mathrm{vs} \\
\text { Negative }\end{array}$} \\
\hline & $\mathrm{n}$ & $\%$ & $\mathrm{n}$ & $\%$ & $\mathrm{n}$ & $\%$ & $\mathrm{n}$ & $\%$ & $\mathrm{n}$ & $\%$ & & & & \\
\hline \multicolumn{15}{|c|}{ Multiple cancer } \\
\hline Yes & 4 & $10.3 \%$ & 39 & $22.5 \%$ & 29 & $14.2 \%$ & 68 & $18.0 \%$ & 279 & $12.6 \%$ & \multirow[b]{2}{*}{0.121} & \multirow{2}{*}{0.618} & \multirow[b]{2}{*}{0.272} & \multirow{2}{*}{0.811} \\
\hline No & 35 & $89.7 \%$ & 134 & $77.5 \%$ & 175 & $85.8 \%$ & 309 & $82.0 \%$ & 1935 & $87.4 \%$ & & & & \\
\hline \multicolumn{15}{|c|}{ Bilateral cancer } \\
\hline Yes & 10 & $27.8 \%$ & 43 & $33.9 \%$ & 54 & $30.2 \%$ & 97 & $31.7 \%$ & 444 & $23.5 \%$ & \multirow{2}{*}{0.550} & \multirow{2}{*}{0.844} & \multirow{2}{*}{0.707} & \multirow{2}{*}{0.553} \\
\hline No & 26 & $72.2 \%$ & 84 & $66.1 \%$ & 125 & $69.8 \%$ & 209 & $68.3 \%$ & 1446 & $76.5 \%$ & & & & \\
\hline \multicolumn{15}{|c|}{ Multiple personal cancers ^ } \\
\hline Breast + Ovarian & 1 & $2.6 \%$ & 25 & $14.5 \%$ & 14 & $6.9 \%$ & 39 & $10.3 \%$ & 59 & $2.7 \%$ & 0.055 & 0.477 & 0.155 & 1.000 \\
\hline $\begin{array}{c}\text { Breast }+ \\
\text { Colorectal } \\
\end{array}$ & 1 & $2.6 \%$ & 2 & $1.2 \%$ & 2 & $1.0 \%$ & 4 & $1.1 \%$ & 18 & $0.8 \%$ & 0.458 & 0.410 & 0.390 & 0.283 \\
\hline $\begin{array}{c}\text { Breast + Corpus } \\
\text { uteri /uterus }\end{array}$ & 1 & $2.6 \%$ & 2 & $1.2 \%$ & 1 & $0.5 \%$ & 3 & $0.8 \%$ & 19 & $0.9 \%$ & 0.458 & 0.296 & 0.326 & 0.296 \\
\hline $\begin{array}{c}\text { Ovarian + } \\
\text { Corpus } \\
\text { uteri/uterus }\end{array}$ & 1 & $2.6 \%$ & 1 & $0.6 \%$ & 2 & $1.0 \%$ & 3 & $0.8 \%$ & 69 & $3.1 \%$ & 0.335 & 0.410 & 0.326 & 1.000 \\
\hline \multicolumn{15}{|c|}{ Family history of cancers in 1 st and 2 nd degree } \\
\hline Breast Cancer & 19 & $48.7 \%$ & 94 & $54.3 \%$ & 128 & $62.7 \%$ & 222 & $58.9 \%$ & 710 & $32.1 \%$ & 0.595 & 0.110 & 0.236 & 0.037 \\
\hline Ovarian Cancer & 0 & $0.0 \%$ & 49 & $28.3 \%$ & 25 & $12.3 \%$ & 74 & $19.6 \%$ & 87 & $3.9 \%$ & $<0.001$ & 0.018 & 0.001 & 0.401 \\
\hline Prostate Cancer & 6 & $15.4 \%$ & 3 & $1.7 \%$ & 24 & $11.8 \%$ & 27 & $7.2 \%$ & 90 & $4.1 \%$ & 0.001 & 0.594 & 0.109 & 0.005 \\
\hline Liver Cancer & 4 & $10.3 \%$ & 19 & $11.0 \%$ & 31 & $15.2 \%$ & 50 & $13.3 \%$ & 234 & $10.6 \%$ & 1.000 & 0.618 & 0.803 & 1.000 \\
\hline Stomach Cancer & 5 & $12.8 \%$ & 29 & $16.8 \%$ & 21 & $10.3 \%$ & 50 & $13.3 \%$ & 169 & $7.6 \%$ & 0.636 & 0.581 & 1.000 & 0.221 \\
\hline Lung Cancer & 6 & $15.4 \%$ & 33 & $19.1 \%$ & 55 & $27.0 \%$ & 88 & $23.3 \%$ & 434 & $19.6 \%$ & 0.819 & 0.159 & 0.318 & 0.684 \\
\hline $\begin{array}{l}\text { Colorectal } \\
\text { Cancer }\end{array}$ & 6 & $15.4 \%$ & 28 & $16.2 \%$ & 37 & $18.1 \%$ & 65 & $17.2 \%$ & 392 & $17.7 \%$ & 1.000 & 0.821 & 1.000 & 0.834 \\
\hline
\end{tabular}

Remarks: cases with double mutation of BRCA1 and PALB2 were excluded from the above table; ${ }^{*} p$-value calculations were limited by sample size; ^ only listed the multiple cancer cases with mutation in PABL2.

Most of the breast tumors were diagnosed with invasive ductal carcinoma (NOS type) (34, $81.0 \%)$ of grade $2(19,59.4 \%)$. Many breast cancers were found to be hormonal positive (ER: 32, 84.2\%; PR: 23, 60.5\%) with negative expressions of HER2 (25, 86.2\%). There were only four $(11.4 \%)$ triple-negative breast cancers (TNBC). The majority of ovarian cancers were diagnosed with epithelial cancer $(3,100 \%)$. Detailed pathological characteristics was shown in Table 3.

There was no significant difference at the age of breast diagnosis and histology between PALB2 mutation carriers and BRCA1/2 mutation carriers. Interestingly, all PALB2 and $B R C A 1$ mutation carriers were female, and $6.9 \%$ of $B R C A 2$ mutation carriers were men (Table 2). Unlike mutation carriers of $B R C A 1 / 2$, ovarian cancer is not commonly seen in PABL2 mutation carriers; $26.6 \%$ of the $B R C A 1$ developed personal ovarian cancer, while only $7.7 \%$ of PABL2 carriers developed ovarian cancer ( $p$-value $<0.001$ ). Both PABL2 and $B R C A 1 / 2$ mutation carriers had strong family histories of breast cancer. A family history of ovarian cancer was only seen in $B R C A 1 / 2$ families but not in PALB2 families, where $28.3 \%$ of $B R C A 1$ carriers and $12.3 \%$ of $B R C A 2$ carriers had a family history of ovarian cancer ( $p$-value $=0.001$ and 0.018 , respectively). Family history of prostate cancer was seen in $15.4 \%$ of PALB2 carriers but in only $1.7 \%$ of $B R C A 1$ carriers ( $p$-value $=0.001$ ); there was no significant different between PALB2 and BRCA2 carriers ( $p$-value $=0.594)$. PALB2 carriers had significant family histories in breast and prostate cancers when comparing with non-carriers ( $p$-value $=0.037$ and 0.005 , respectively). Association of age of ovarian diagnosis and histology between PALB2 mutation carriers and BRCA1/2 mutation carriers was not calculated due to limited numbers.

\subsection{Double Heterozygote in BRCA1 and PALB2}

Double mutations were identified in a 63-year-old female diagnosed with bilateral invasive ductal breast carcinoma of grade 2 and 3, both ER- at the age of 30 and 32 years. She was also diagnosed with FIGO grade 3 epithelial serous ovarian cancer at the age of 48 and cancer at the glottis larynx of T1N0M0 at the age of 58. Detailed family pedigree is shown in Figure 1. 


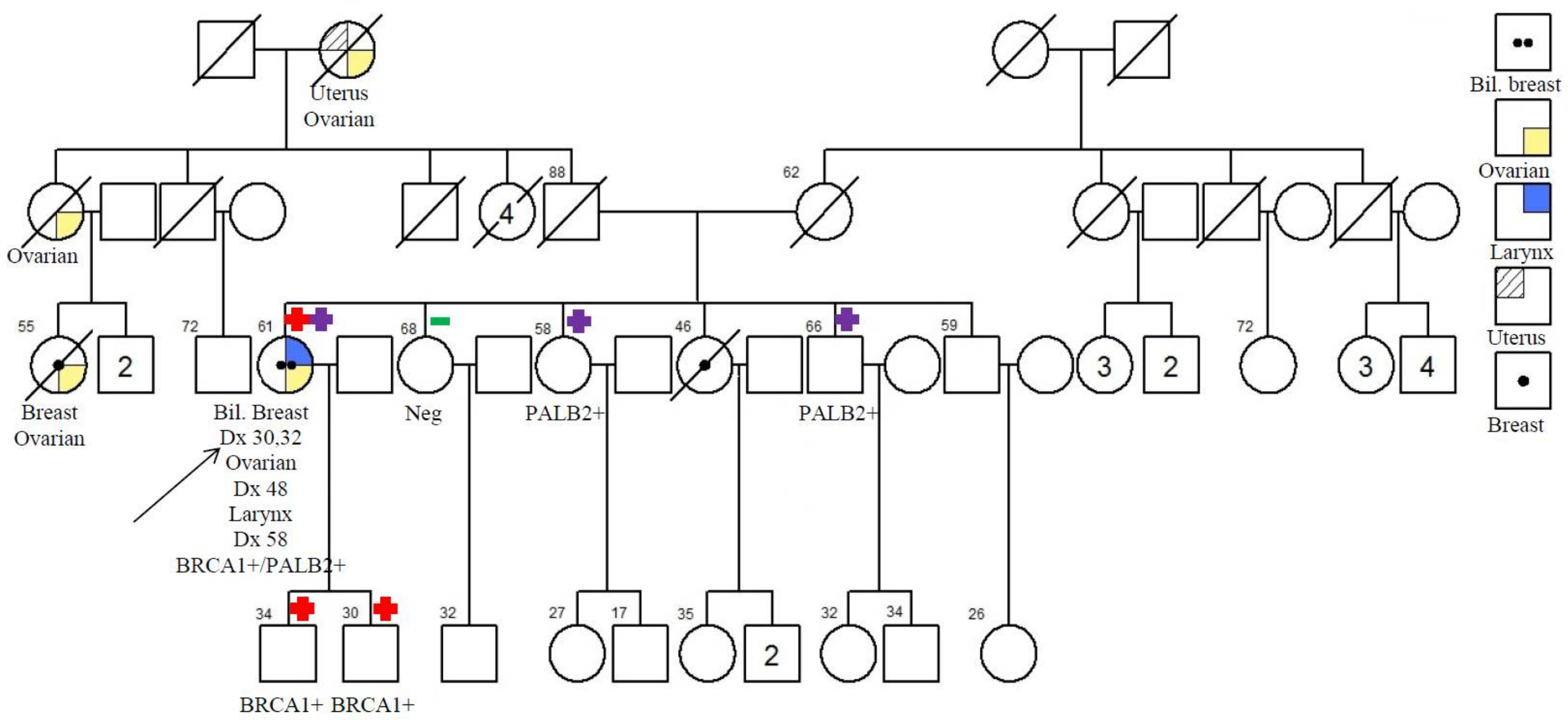

Figure 1. Pedigree of the proband's family with double heterozygote in BRCA1 and PALB2. BRCA1 carrier; PALB2 carrier; - negative; Bil. Breast: bilateral breast. 
Loss of function variants in $B R C A 1$ c.3286C $>$ T; p.Gln1096* and a deletion that resulted in frameshift termination c.857delC; p.Pro286Leufs*2 in PALB2 were identified in this patient (not listed in Figure 2 and Table 3). Mutation c.3286C $>$ T; p.Gln1096* was a base pair alternation in exon 11-9/10 of $B R C A 1$, which is not located in any of the reported domains. c.857delC; p.Pro286Leufs*2 in PALB2 was located in exon 4-3.

Table 3. Pathological characteristics and mutation frequencies of PALB2 and BRCA1/2.

\begin{tabular}{|c|c|c|c|c|c|c|c|c|c|c|c|c|c|c|}
\hline \multirow[t]{3}{*}{$\begin{array}{l}\text { No. of } \\
\text { Tumors }\end{array}$} & \multirow{2}{*}{\multicolumn{2}{|c|}{$\begin{array}{c}n=46 \\
P A L B 2+\end{array}$}} & \multirow{2}{*}{\multicolumn{2}{|c|}{$\begin{array}{c}n=170 \\
B R C A 1+\end{array}$}} & \multirow{2}{*}{\multicolumn{2}{|c|}{$\begin{array}{c}n=233 \\
B R C A 2+\end{array}$}} & \multirow{2}{*}{\multicolumn{2}{|c|}{$\begin{array}{l}n=403 \\
B R C A+\end{array}$}} & \multirow{2}{*}{\multicolumn{2}{|c|}{$\begin{array}{c}n=2234 \\
\text { Negative }\end{array}$}} & \multicolumn{4}{|c|}{$p$-Value } \\
\hline & & & & & & & & & & & \multirow{2}{*}{$\begin{array}{l}\text { PALB2+ } \\
\text { vs. } \\
\text { BRCA1+ }\end{array}$} & \multirow{2}{*}{$\begin{array}{l}\text { PALB2+ } \\
\text { vs. } \\
\text { BRCA2+ }\end{array}$} & \multirow{2}{*}{$\begin{array}{c}\text { PALB2+ } \\
\text { vs. } \\
\text { BRCA+ }\end{array}$} & \multirow{2}{*}{$\begin{array}{c}P A L B 2+\text { vs. } \\
\text { Negative }\end{array}$} \\
\hline & $\mathbf{n}$ & $\%$ & $\mathbf{n}$ & $\%$ & $\mathbf{n}$ & $\%$ & $\mathbf{n}$ & $\%$ & $\mathbf{n}$ & $\%$ & & & & \\
\hline \multicolumn{15}{|c|}{ Breast histology } \\
\hline Ductal & 34 & $81.0 \%$ & 132 & $80.0 \%$ & 156 & $69.0 \%$ & 288 & $73.7 \%$ & 1548 & $68.8 \%$ & \multirow{3}{*}{0.124} & \multirow{3}{*}{0.369} & \multirow{3}{*}{0.507} & \multirow{3}{*}{0.305} \\
\hline In situ & 5 & $11.9 \%$ & 8 & $4.8 \%$ & 40 & $17.7 \%$ & 48 & $12.3 \%$ & 412 & $18.3 \%$ & & & & \\
\hline Other & 3 & $7.1 \%$ & 25 & $15.2 \%$ & 30 & $13.3 \%$ & 55 & $14.1 \%$ & 291 & $12.9 \%$ & & & & \\
\hline \multicolumn{15}{|c|}{ Grade (invasive grade) } \\
\hline 1 & 1 & $3.1 \%$ & 5 & $3.9 \%$ & 6 & $4.1 \%$ & 11 & $4.0 \%$ & 289 & $18.4 \%$ & \multirow{3}{*}{$<0.001$} & \multirow{3}{*}{0.779} & \multirow{3}{*}{0.067} & \multirow{3}{*}{0.048} \\
\hline 2 & 19 & $59.4 \%$ & 30 & $23.3 \%$ & 75 & $51.0 \%$ & 105 & $38.0 \%$ & 733 & $46.7 \%$ & & & & \\
\hline 3 & 12 & $37.5 \%$ & 94 & $72.9 \%$ & 66 & $44.9 \%$ & 160 & $58.0 \%$ & 549 & $34.9 \%$ & & & & \\
\hline \multicolumn{15}{|c|}{ ER } \\
\hline Positive & 32 & $84.2 \%$ & 46 & $29.1 \%$ & 178 & $84.8 \%$ & 224 & $60.9 \%$ & 1517 & $71.9 \%$ & \multirow{2}{*}{$<0.001$} & \multirow{2}{*}{1.000} & & \\
\hline Negative & 6 & $15.8 \%$ & 112 & $70.9 \%$ & 32 & $15.2 \%$ & 144 & $39.1 \%$ & 592 & $28.1 \%$ & & & 0.004 & 0.103 \\
\hline & & & & & & & & $\mathrm{R}$ & & & & & & \\
\hline Positive & 23 & $60.5 \%$ & 29 & $18.8 \%$ & 141 & $68.4 \%$ & 170 & $47.2 \%$ & 1250 & $60.0 \%$ & & & & \\
\hline Negative & 15 & $39.5 \%$ & 125 & $81.2 \%$ & 65 & $31.6 \%$ & 190 & $52.8 \%$ & 832 & $40.0 \%$ & $<0.001$ & 0.352 & 0.128 & 1.000 \\
\hline & & & & & & & & ER2 & & & & & & \\
\hline Positive & 4 & $13.8 \%$ & 9 & $6.6 \%$ & 36 & $20.6 \%$ & 45 & $14.4 \%$ & 456 & $25.5 \%$ & & & & \\
\hline Negative & 25 & $86.2 \%$ & 128 & $93.4 \%$ & 139 & $79.4 \%$ & 267 & $85.6 \%$ & 1331 & $74.5 \%$ & 0.246 & 0.461 & 1.000 & 0.197 \\
\hline & & & & & & Molecu & subtyl & invasive & umor or & & & & & \\
\hline TNBC & 4 & $11.4 \%$ & 91 & $60.7 \%$ & 21 & $11.5 \%$ & 112 & $33.6 \%$ & 314 & $17.6 \%$ & & & & \\
\hline Her2+ & 1 & $2.9 \%$ & 4 & $2.7 \%$ & 5 & $2.7 \%$ & 9 & $2.7 \%$ & 152 & $8.5 \%$ & $<0.001$ & 0.730 & 0.027 & 0.338 \\
\hline ER/PR+ & 29 & $82.9 \%$ & 46 & $30.7 \%$ & 155 & $84.7 \%$ & 201 & $60.4 \%$ & 1295 & $72.5 \%$ & & & & \\
\hline & & & & & & & & age & & & & & & \\
\hline 0 & 3 & $7.7 \%$ & 15 & $9.2 \%$ & 41 & $19.0 \%$ & 56 & $14.8 \%$ & 424 & $19.5 \%$ & & & & \\
\hline 1 & 13 & $33.3 \%$ & 77 & $47.2 \%$ & 68 & $31.5 \%$ & 145 & $38.3 \%$ & 795 & $36.6 \%$ & & & & \\
\hline 2 & 18 & $46.2 \%$ & 60 & $36.8 \%$ & 67 & $31.0 \%$ & 127 & $33.5 \%$ & 620 & $28.5 \%$ & 0.160 & 0.145 & 0.270 & 0.104 \\
\hline 3 & 3 & $7.7 \%$ & 10 & $6.1 \%$ & 33 & $15.3 \%$ & 43 & $11.3 \%$ & 243 & $11.2 \%$ & & & & \\
\hline 4 & 2 & $5.1 \%$ & 1 & $0.6 \%$ & 7 & $3.2 \%$ & 8 & $2.1 \%$ & 90 & $4.1 \%$ & & & & \\
\hline & & & & & & & Ovar & histolog & & & & & & \\
\hline Epithelial & 3 & $100.0 \%$ & 76 & $100.0 \%$ & 36 & $97.3 \%$ & 112 & $99.1 \%$ & 355 & $95.4 \%$ & & & & \\
\hline Germ cell & 0 & $0.0 \%$ & 0 & $0.0 \%$ & 0 & $0.0 \%$ & 0 & $0.0 \%$ & 7 & $1.9 \%$ & & & & \\
\hline Stromal & 0 & $0.0 \%$ & 0 & $0.0 \%$ & 0 & $0.0 \%$ & 0 & $0.0 \%$ & 5 & $1.3 \%$ & $\mathrm{NA}^{*}$ & $\mathrm{NA}^{*}$ & $\mathrm{NA}^{*}$ & $\mathrm{NA}^{*}$ \\
\hline Mixed & 0 & $0.0 \%$ & 0 & $0.0 \%$ & 1 & $2.7 \%$ & 1 & $0.9 \%$ & 5 & $1.3 \%$ & & & & \\
\hline Not stated & 2 & - & 4 & - & 4 & - & 8 & - & 27 & - & & & & \\
\hline
\end{tabular}

Remarks: cases with double mutation of BRCA1 and $P A L B 2$ were excluded from above calculation; * $p$-value calculations were limited by sample size.

\subsection{Mutations Specific in PALB2}

In this cohort, 39 mutations (1.5\%) were identified with 24 types of mutation variants. Eleven $(45.8 \%)$ of the mutation variants involving a frame-shift resulted in early termination, nine $(37.5 \%)$ were nonsense mutations, three $(12.5 \%)$ of the mutations happened at splice sites, and one (4.2\%) was a large deletion of exon 4-6 (Table 4). There were seven novel mutations identified (c.181C>T; p.Gln61*, c.212-712_2587-888de19762; p.Pro72Serfs*20, c.448C > T; p.Gln150*, c.1038delA; p.Glu347Asnfs*9, c.1914dupT; p.Glu639*, c.2016dupA; 
p.Glu673Argfs*42 and c.3201+2T>C; r.3114_3350del237; p.Asn1039_Arg1117del79). There was no specific genomic regional clustering for these mutations in PALB2. However, the most frequent mutations (c.2108T>G; p.Leu703* and c.1059delA; p.Lys353Asnfs*3) were seen in seven $(17.9 \%)$ and five $(12.8 \%)$ unrelated families, respectively. Details of these mutation variants are shown in Figure 2 and Table 4.

Table 4. Germline heterozygous variants identified in PALB2.

\begin{tabular}{|c|c|c|c|}
\hline HVGS & Frequency & Type of Mutation & $\begin{array}{l}\text { Novel/ } \\
\text { Reference }\end{array}$ \\
\hline c.15delC; p.Lys7Serfs*11 & 1 & Frameshift termination & [18] \\
\hline c. $181 C>T ;$ p.Gln61* & 1 & Nonsense & Yes \\
\hline c. $211+1 G>A$ & 3 & Splice site mutation & [19] \\
\hline $\begin{array}{c}\text { Deletion exon 4-6; c.212-712_2587-888del9762; } \\
\text { p.Pro72Serfs }{ }^{* 20}\end{array}$ & 1 & Large deletion & Yes \\
\hline c.444delG; p.Lys149Serfs*28 & 1 & Frameshift termination & {$[20]$} \\
\hline c. $448 \mathrm{C}>\mathrm{T} ;$ p.G $\ln 150^{*}$ & 1 & Nonsense & Yes \\
\hline c.839delA; p.Asn280Thrfs*8 & 1 & Frameshift termination & [21] \\
\hline c.1038delA; p.Glu347Asnfs*9 & 1 & Frameshift termination & Yes \\
\hline c. $1048 \mathrm{C}>\mathrm{T} ;$ p.G $\ln 350^{*}$ & 1 & Nonsense & {$[22]$} \\
\hline c.1059delA; p.Lys353Asnfs*3 & 5 & Frameshift termination & [23] \\
\hline c. $1240 \mathrm{C}>\mathrm{T} ; \mathrm{p} . \operatorname{Arg} 414^{*}$ & 1 & Nonsense & [24] \\
\hline c.1446delC; p.Ser483Hisfs*2 & 1 & Frameshift termination & {$[18]$} \\
\hline c.1592delT; p.Leu531Cysfs*30 & 2 & Frameshift termination & [25] \\
\hline c.1914dupT; p.Glu639* & 1 & Nonsense & Yes \\
\hline c.2016dupA; p.Glu673Argfs*42 & 1 & Frameshift termination & Yes \\
\hline c.2108T>G; p.Leu703* & 7 & Nonsense & [26] \\
\hline c.2219_2220delAA; p.Gln740Argfs*4 & 1 & Frameshift termination & [27] \\
\hline c.2594C>G; p.Ser865* & 1 & Nonsense & [28] \\
\hline c.2760dupA; p.Gln921Thrfs*7 & 1 & Frameshift termination & [29] \\
\hline c.2968G>T; p.Glu990* & 3 & Nonsense & [30] \\
\hline $\begin{array}{l}\text { c.3114-1G>A; r.3114_3350del237; } \\
\text { p.Asn1039_Arg1117del79 }\end{array}$ & 1 & Splice site mutation & [31] \\
\hline $\begin{array}{l}\text { c.3201+2T>C; r.3114_3350del237; } \\
\text { p.Asn1039_Arg1117del79 }\end{array}$ & 1 & Splice site mutation & Yes \\
\hline c.3256C>T; p.Arg1086* & 1 & Nonsense & [32] \\
\hline c.3507_3508delTC; p.His1170Phefs*19 & 1 & Frameshift termination & [33] \\
\hline
\end{tabular}

Classification and regression tree modelling were performed on the clinicopathological variables to predict the probability of harboring PALB2 and BRCA1 mutations. Patients with the PALB2 mutation were most likely to have personal breast cancer only and no family history of prostate and ovarian cancer when comparing with BRCA1 carriers (Figure 3). The modelling showed no stratification between PALB2 and BRCA2 carriers, which further confirmed that PALB2 was the partner and localizer of BRCA2. As shown in Figure 4, $B R C A 1 / 2$ carriers were more likely to have family history of ovarian cancer, but this was not seen in the PALB2 families. The association with a family history of prostate cancer was seen in PALB2 carriers compared with mutation negative patients (Figure 4). 


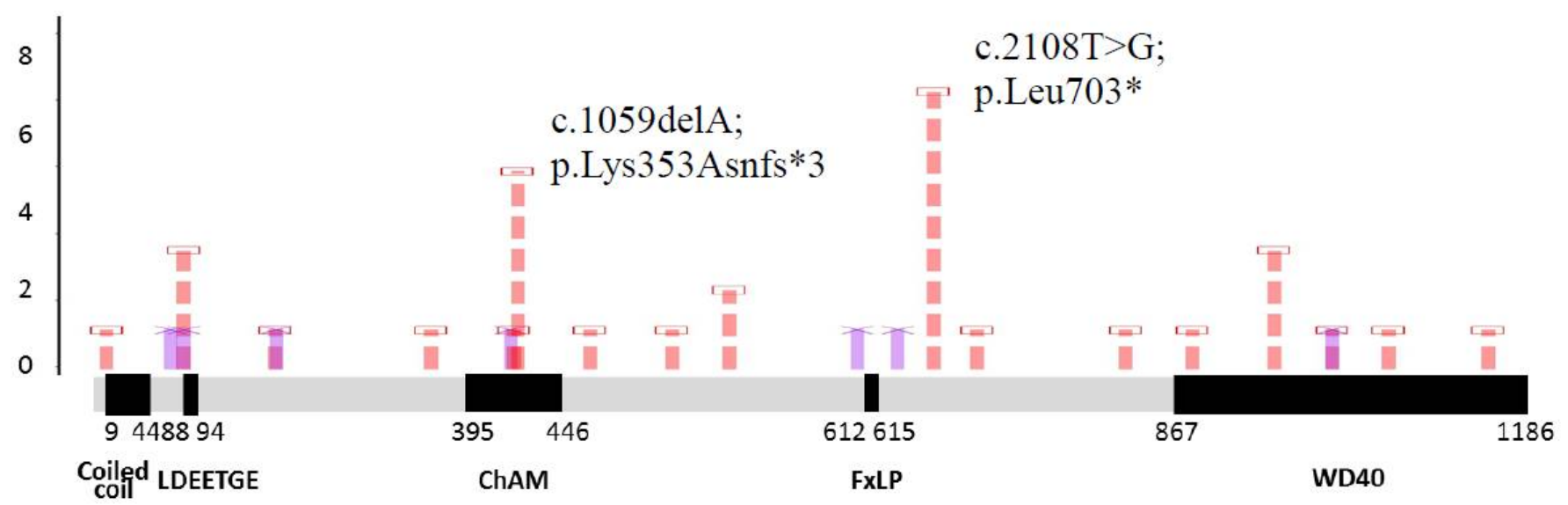

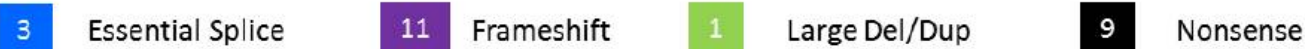

Figure 2. Mutations found in PALB2. ChAM: chromatin association motif; LDEETGE: extended EDGE motif; II novel mutation;

There was no significant difference in disease-free survival probability or overall survival between PALB2 mutation breast carriers and the mutation-negative breast cancer group, as well as to those with the BRCA1/2 mutation group in pairwise comparisons (Supplementary Figures S1 and S2).

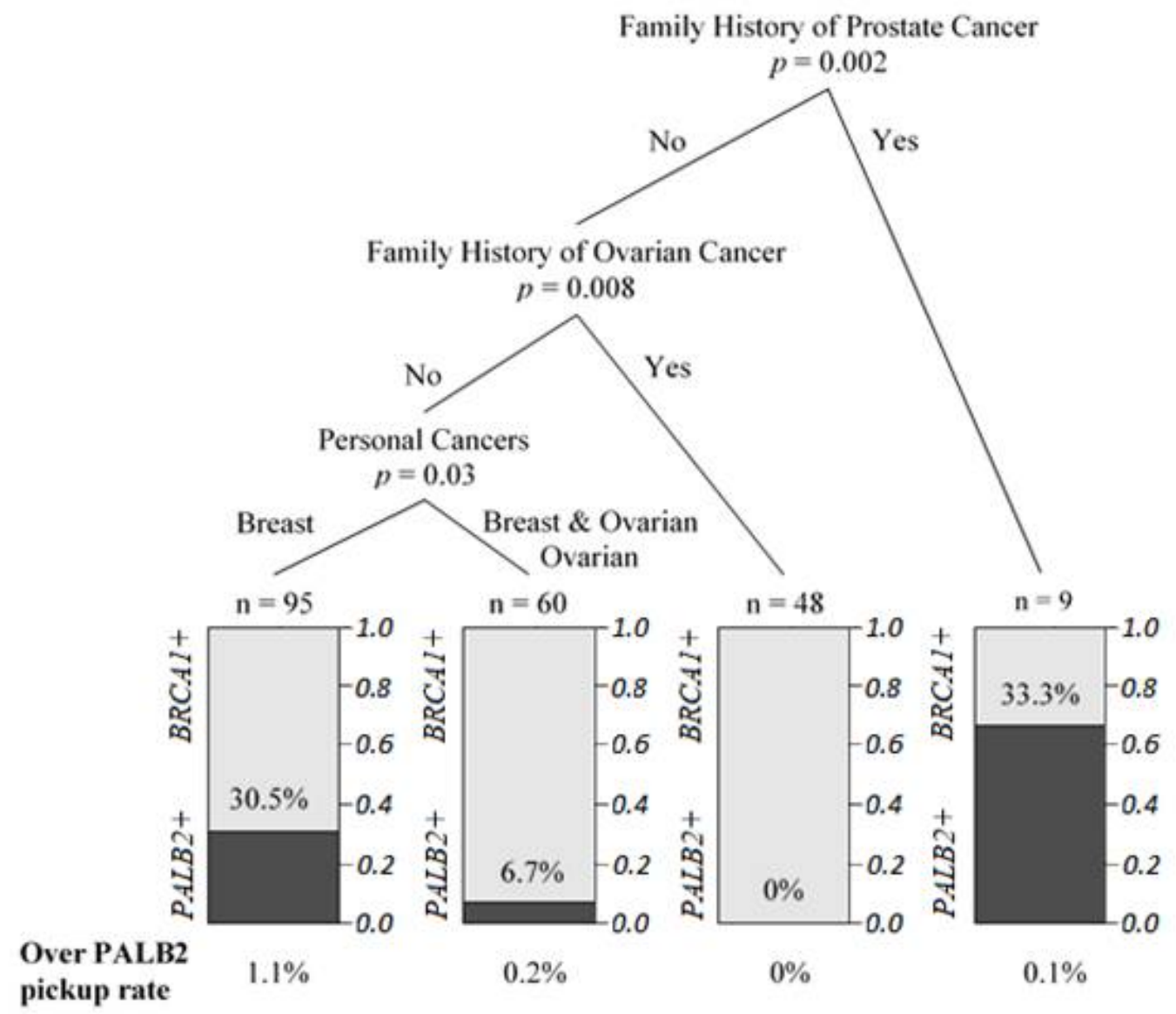

Figure 3. Regression tree for prediction of probability to find PALB2 pathogenic mutation carriers from $B R C A 1$ carriers. 


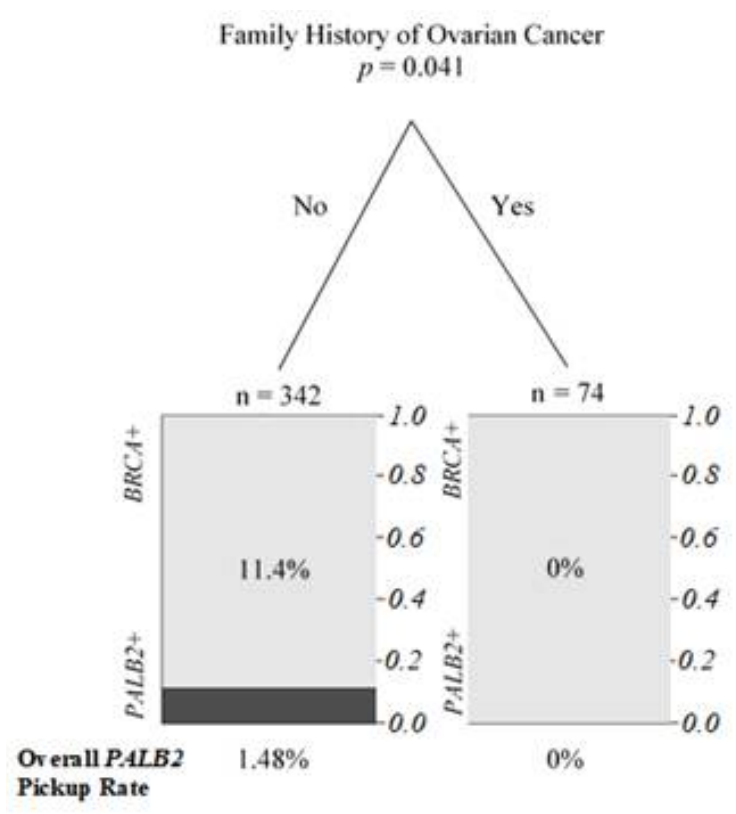

(A)

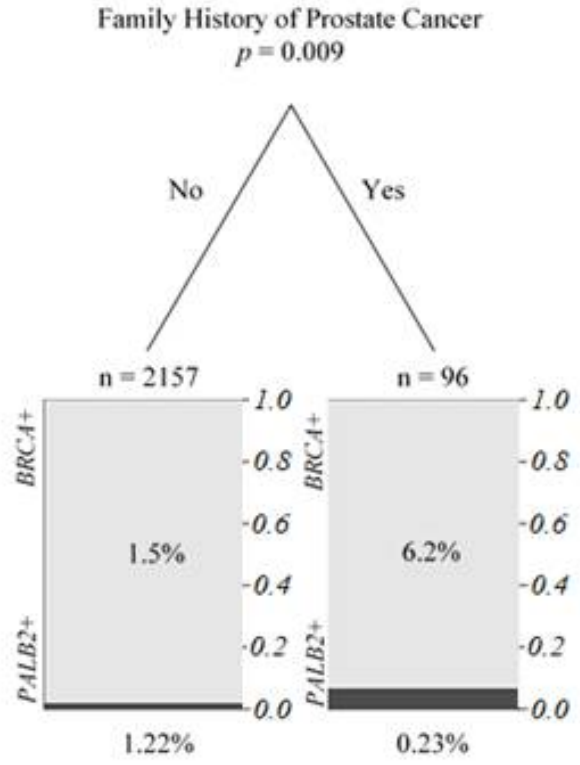

(B)

Figure 4. Regression tree for prediction of probability to find PALB2 pathogenic mutation carriers. (A) Regression tree to identify $P A B L 2$ pathogenic carriers from $B R C A 1 / 2$ carriers. (B) Regression tree to identify PABL2 pathogenic carriers from mutation negative non-carriers.

\section{Discussion}

PALB2 has been identified as the third most prevalent breast cancer causing gene with characteristics attributable to germline loss of function monoallelic mutations [6]. According to studies performed in different populations, PALB2 mutation frequencies in familial breast cancer cases range between 0.6 and $2.7 \%[1,10,15]$, while the cumulative average risk reaches approximately $35 \%$ by the age of 70 , which is similar to the cumulate average risk conferred by germline $B R C A 2$ mutations [1]. PALB2 mutation frequency in a Polish cohort was found to be $0.93 \%$ [34], $0.8 \%$ in the nationwide United States' population [35], and $0.97 \%$ in a Chinese cohort in Mainland China [36].

PALB2 was believed to contribute moderately to breast cancer risk previously. However, recent studies have identified that the cancer risk is similar to that of $B R C A 2 \mathrm{mu}-$ tations [1]. Family members of patients carrying familial PALB2 mutations have a 79.5-fold increased risk of developing breast cancer with the highest predisposing age group below 40 years [1,3]. A panel study on 34 breast cancer predisposition genes in hereditary breast and ovarian cancer (HBOC) families revealed that the odds ratio of PALB2 was $10.25(\mathrm{CI}=6.03-16.40)$, which was similar to the odds ratio of $B R C A 2$, namely $10.26(\mathrm{CI}=5.75-16.33)$, therefore deeming it as a high penetrance gene $[37,38]$. The risk of breast cancer for PALB2 mutation carriers has been studied worldwide in various populations; the risk of developing breast cancer was six times higher $(\mathrm{CI}=2.2-17.2$; $p=0.01$ ) in Finnish families carrying the c.1592delT founder mutation as compared to the risk of developing non-breast cancer, which was $1.4(\mathrm{CI}=0.6-3.2 ; p=0.5)$ times against normal individuals [39]. In another study performed in a Polish population, two recurrent PALB2 mutations, c.172_175delGA and c.509_510delGA, were identified at a frequency of $1.5 \%$ [40]. Similarly, another PALB2 pathogenic variant, c. $2323 \mathrm{C}>\mathrm{T}$ was identified in French-Canadian women in Quebec, where the Q775X mutation occurred in about 0.5\% of the women diagnosed for breast cancer [41]. An Asian study identified $0.73 \%$ and $0.14 \%$ in breast cancer and normal control cohorts, respectively, and the most common recurrent mutations were c.7G $>\mathrm{T}$ and c.2968G $>\mathrm{T}$ in the cancer cohort [19]. A higher risk of developing breast cancer was estimated in PALB2 mutation carriers, which was 9.1-fold compared to non-carriers in the UK [1]. Another study showed an odds ratio of 2.3 in 
Australian patients carrying PALB2 truncating mutations [12]. The absolute risk of breast cancer and male breast cancer were $52.8 \%$ and $0.9 \%$, respectively, by age of 80 [3].

Interestingly, we identified a patient with double heterozygous PALB2 c.857delC and $B R C A 1$ c.3286C $>$ T mutations who was diagnosed with bilateral breast, ovarian, and larynx cancers. She underwent mastectomy on her right side breast and modified radical mastectomy on her left side breast due to her early age. She also underwent bilateral salpingo-oophorectomy and received chemotherapy, which was well tolerated. She has shown no signs of recurrence after treatment. In the family study, we were only able to obtain the genetic analyses of five of her unaffected cancer-free family members. Her brother and sister both carried only one of the two mutations, namely PALB2 c.857delC. Both of her sons carried another BRCA1 c.3286C $>$ T mutation. These findings show that the two mutations are segregated independently (Figure 1). Double heterozygous PALB2 c.758insT and BRCA1 c.927delA mutations have also been identified in a German patient with triple-negative breast cancer. She also presented with large myomas of the uterus, a small meningioma and bipolar disorder. However, she has a strong familial aggregation of breast cancer [42]. Another French-Canadian woman with breast cancer was found to harbor PALB2 c.2323C $>$ T and BRCA2 c. $9004 \mathrm{G}>\mathrm{A}$. The maternal side of the double heterozygous carrier contributed to Lynch Syndrome [43].

PALB2 mutations have been shown to be associated with FA, and patients with FA are sensitive to cross-linking agents [44]. A phase II clinical trial of talazoparib was performed on patients with HER2- breast cancer or other tumors with mutated HR pathway genes, which showed a RECIST response in patients carrying PALB2 mutations [45]. Similarly, patients with HER2- metastatic breast cancer who received olaparib had an objective response rate of $82 \%$ [46]. One patient with two deleterious PALB2 mutations, one in exon 4 , c.1653T>A, and another in exon 6, c.2576C $>\mathrm{G}$, was administered with olaparib and showed a 70\% reduction in the tumor burden [47]. Another clinical trial (NCT04756765) based on the use of talazoparib on advanced PALB2 mutation associated breast cancer is currently underway [48]. Survival data has been studied in different populations, among which Chinese and Polish populations had hazard ratios of $8.38(\mathrm{CI}=2.19-32.11, p=0.002)$ and $2.27(\mathrm{CI}=1.64-3.15 ; p<0.0001)$, respectively, thereby with poor overall survival $[10,17]$. Overall, PALB2 mutation carriers with breast cancer had a poor 10-year survival rate of $48 \%$ with tumors $<2 \mathrm{~cm}$, while it dropped to $32 \%$ for tumors $>2 \mathrm{~cm}$ in diameter [10].

Evidence supports that the PALB2 mutation confers moderate to high breast cancer risk and increased risk for ovarian and pancreatic cancer. The National Comprehensive Cancer Network (NCCN) guidelines (Version 3.2021) has proposed the management of patients with PALB2 mutations, which includes two strategies [49]. The first is an annual mammogram or consideration of a breast MRI by the age of 30, which may be adjusted to 5-10 years earlier based on the youngest case in the family. Secondly, risk-reducing mastectomy may be performed with respect to residual breast cancer risk and family history. The American College of Medical Genetics and Genomics (ACMG) recommends that PALB2 gene testing should be included in the test panel for breast, ovarian, and pancreatic cancer. In addition, patients with PALB2 mutation should be offered breast cancer surveillance similar to that for $B R C A 1 / 2$ carriers [50]. Currently, there is no standardized therapy for PALB2 mutation associated breast cancer due to the limited amount of evidence available; however, some clinical trials have proved the use of PARP inhibitors as beneficial.

The PALB2 mutation has rarely been found in ovarian cancer patients. The PALB2 mutation was shown to have an increased risk of ovarian cancer with an odds ratio of 4.6 in a European study. The mutation frequency in epithelial ovarian cancer patients was $0.21 \%$ comparing to $0.05 \%$ in the control group [51]. In a systematic review of pathogenic PALB2 mutations, $92.5 \%$ of cases described were breast cancer patients, $5.0 \%$ of cases were ovarian cancer patients, and $2.4 \%$ of cases were pancreatic cancer patients [52]. 


\section{Materials and Methods}

\subsection{Ethics Statement}

This study was approved by the Institutional Review Board of the University of Hong Kong/Hospital Authority West Cluster and respective authorities of other contributing hospitals in Hong Kong. All participants recruited consented to the study, and the research was conducted according to the Declaration of Helsinki.

\subsection{Patients}

A cohort of 2631 patients with breast and/or ovarian cancer were recruited by the Hong Kong Hereditary and High-risk Breast Cancer Registry between 2012 and 2019 with the following selection criteria: (1) had at least one first- or second-degree relative with $B R C A$-associated cancer, regardless of age; (2) the age at breast cancer diagnosis was under 45 years; (3) bilateral breast cancer; (4) triple-negative breast cancers, (5) cancers with medullary type histology; (6) known to be BRCA mutation related family; (7) male breast cancer.

Clinicopathologic characteristics of patient were obtained by medical personnel and medical records (Table 1). Specimens with known BRCA1/2 mutations (positive control) and anonymous normal volunteer local individuals (negative control) were included for validation of the next generation sequencing (NGS) and to evaluate the performance characteristics of NGS.

\subsection{Six-Gene Sequencing Panel}

Genomic DNA samples were extracted from peripheral blood samples and subjected to a 6-gene panel (BRCA1, BRCA2, TP53, PTEN, PALB2 and CDH1) using our previouslydeveloped protocol [53]. Sequencing libraries from the above-mentioned panels were synthesized and purified as previously described [53,54]. Pooled libraries were loaded onto the MiSeq instrument (Illumina, San Diego, CA) for sequencing. The presence of multi-exonic copy number variations (CNVs) was investigated by computational analysis and multiplex ligation-dependent probe amplification (MLPA). The cDNA from blood was also sequenced in the case of splicing variant analysis at the transcript level. All detected pathogenic variants were verified by the conventional Sanger bi-directional DNA sequencing. The sequencing data was co-analyzed by our in-house developed bioinformatics pipeline.

\subsection{Variant Interpretation and Annotation}

Variants calling bioinformatics was performed as previously described [53,54]. Paired sequencing reads were mapped to the human reference genome sequence GRCh37/hg19. The variants with minor allele frequency of at least $1 \%$ reported by the 1000 Genomes Projects [55] were excluded from manual variant curation. Variants were described according to the recommendations of the Human Genome Variation Society (HGVS). Variant descriptions were further verified with Mutalyzer Name Checker (http:/ / mutalyzer.nl, accessed on December 2012 to December 2020).

\subsection{Statistical Analysis}

Clinicopathological variables from pathogenic/likely pathogenic mutation carriers and non-carriers were tabulated in contingency tables. Computation was performed using $\mathrm{R}$ (version 3.6.0). Statistical tests suitable for categorical data were then considered. Some variables had expected values of less than 5 , and most variables did not have a natural ordering. Fisher's exact test was adopted. Bonferroni correction was adopted to adjust the significance level for multiple comparison. Conditional Inference Tree (in R package party kit) was also applied to obtain significant factors to predict mutation. Diseasefree survival analysis was done on breast cancer patients (mutation carriers of 30 PALB2, $93 B R C A 1,158 B R C A 2$, and 1695 non-carriers) with surgical information and follow-up data. The survival time was calculated from the date of the first surgery. The next event was defined as the earliest local relapse, distant metastasis, death, or occurrence of 2nd 
primary cancer. For bilateral cases, if the onset time of 2nd primary was $>90$ days after 1 st primary and before any relapse of 1st primary, the case was treated as censored at the time of 2nd primary diagnosis. The by-gene survival times were then plotted for mutation carriers of 33 PALB2, 93 BRCA1, 163 BRCA2, and 1782 non-carriers. The log-rank test was tested on the significant differences in survival probability. Significance level was set at $5 \%$ ( $p$-value $<0.05)$. Disease-free survival analysis and overall survival in ovarian cancer patients were not included due to the limited number of cases.

\section{Conclusions}

We demonstrated that the mutation frequency of PALB2 was $1.6 \%$ among high-risk breast and $1 \%$ of ovarian cancer patients. We identified seven novel mutations and two recurrent mutations in PALB2. The majority of the PALB2 mutation tumors were found to be hormonal positive and were likely to have familial aggregation of breast cancer. The mutation screening for PALB2 should be included in the test panel for breast and ovarian cancer patients. More clinical evidence is needed to demonstrate the effectiveness of PARP inhibitors in patients with PALB2 mutation, and yet breast cancer surveillance is recommended for these mutation carriers.

Supplementary Materials: The following are available online at https://www.mdpi.com/article/ 10.3390/cancers13164195/s1, Supplementary Figure S1a. Disease-free survival of PALB2 mutation carriers versus BRCA1 mutation carriers. Supplementary Figure S1b. Disease-free survival of PALB2 mutation carriers versus mutation BRCA2 mutation carriers. Supplementary Figure S1c. Disease-free survival of PALB2 mutation carriers versus negative mutation non-carriers. Supplementary Figure S2a. Overall survival of PALB2 mutation carriers versus BRCA1 mutation carriers. Supplementary Figure S2b. Overall survival of PALB2 mutation carriers versus BRCA2 mutation carriers. Supplementary Figure S2c. Overall survival of PALB2 mutation carriers versus mutation negative non-carriers.

Author Contributions: The study was designed by A.K. (Ava Kwong), V.Y.S., T.-L.C., K.K.L.C., H.Y.S.N. and E.S.K.M., C.Y.S.H. performed the experiments and collected data. A.K. (Aleena Khalid) and V.Y.S. drafted the manuscript. C.H.A. performed the bioinformatics analysis. A.K. (Ava Kwong), V.Y.S., T.-L.C., K.K.L.C., H.Y.S.N. and E.S.K.M. reviewed the manuscript. All authors have read and agreed to the published version of the manuscript.

Funding: This study was supported by Dr. Ellen Li Charitable Foundation; Kerry Kuok Foundation; Health and Medical Research Fund (03143406); Asian Fund for Cancer Research; and Hong Kong Hereditary Breast Cancer Family Registry.

Institutional Review Board Statement: The study was conducted according to the guidelines of the Declaration of Helsinki and approved by the Institutional Review Board of the University of Hong Kong/Hospital Authority West Cluster and respective authorities of other contributing hospitals in Hong Kong (protocol code UW 06-274 T1299).

Informed Consent Statement: Written informed consent was obtained from participants recruited in this study.

Data Availability Statement: The dataset supporting the conclusions of this article is included within the article and its additional files. Mutated sequences (except those mutations of structural rearrangement, which required further investigation) have been submitted to Genbank.

Acknowledgments: We thank Dona Ho and Elaine Wong from Hong Kong Sanatorium \& Hospital for assistance with processing sequencing data. Luke Luk and Terry Fung assisted in statistical analysis.

Conflicts of Interest: The authors declare that they have no competing interests. 


\section{References}

1. Antoniou, A.C.; Casadei, S.; Heikkinen, T.; Barrowdale, D.; Pylkäs, K.; Roberts, J.; Lee, A.; Subramanian, D.; De Leeneer, K.; Fostira, F.; et al. Breast cancer risk in families with mutations in PALB2. N. Engl. J. Med. 2014, 371, 497-506. [CrossRef]

2. Cao, A.Y.; Huang, J.; Hu, Z.; Li, W.F.; Ma, Z.L.; Tang, L.L.; Zhang, B.; Su, F.X.; Zhou, J.; Di, G.H.; et al. The prevalence of PALB2 germline mutations in BRCA1/BRCA2 negative Chinese women with early onset breast cancer or affected relatives. Breast Cancer Res. Treat. 2009, 114, 457-462. [CrossRef]

3. Yang, X.; Leslie, G.; Doroszuk, A.; Schneider, S.; Allen, J.; Decker, B.; Dunning, A.M.; Redman, J.; Scarth, J.; Plaskocinska, I.; et al. Cancer Risks Associated with Germline PALB2 Pathogenic Variants: An International Study of 524 Families. J. Clin. Oncol. 2020, 38, 674-685. [CrossRef]

4. $\quad$ Erkko, H.; Xia, B.; Nikkilä, J.; Schleutker, J.; Syrjäkoski, K.; Mannermaa, A.; Kallioniemi, A.; Pylkäs, K.; Karppinen, S.M.; Rapakko, K.; et al. A recurrent mutation in PALB2 in Finnish cancer families. Nature 2007, 446, 316-319. [CrossRef] [PubMed]

5. Ece Solmaz, A.; Yeniay, L.; Gökmen, E.; Zekioğlu, O.; Haydaroğlu, A.; Bilgen, I.; Özkınay, F.; Onay, H. Clinical Contribution of Next-Generation Sequencing Multigene Panel Testing for BRCA Negative High-Risk Patients with Breast Cancer. Clin Breast Cancer. 2021, 12. [CrossRef]

6. Evans, M.K.; Longo, D.L. PALB2 mutations and breast-cancer risk. N. Engl. J. Med. 2014, 371, 566-568. [CrossRef]

7. Slater, E.P.; Langer, P.; Niemczyk, E.; Strauch, K.; Butler, J.; Habbe, N. PALB2 mutations in European familial pancreatic cancer families. Clin. Genet. 2010, 78, 490-494. [CrossRef]

8. Reid, S.; Schindler, D.; Hanenberg, H.; Barker, K.; Hanks, S.; Kalb, R.; Neveling, K.; Kelly, P.; Seal, S.; Freund, M.; et al. Biallelic mutations in PALB2 cause Fanconi anemia subtype FA-N and predispose to childhood cancer. Nat. Genet. 2007, 39, 162-164. [CrossRef] [PubMed]

9. Tischkowitz, M.; Xia, B.; Sabbaghian, N.; Reis-Filho, J.S.; Hamel, N.; Li, G.; van Beers, E.H.; Li, L.; Khalil, T.; Quenneville, L.A.; et al. Analysis of PALB2/FANCN-associated breast cancer families. Proc. Natl. Acad. Sci. USA 2007, 104, 6788-6793. [CrossRef] [PubMed]

10. Deng, M.; Chen, H.H.; Zhu, X.; Luo, M.; Zhang, K.; Xu, C.J.; Hu, K.M.; Cheng, P.; Zhou, J.J.; Zheng, S.; et al. Prevalence and clinical outcomes of germline mutations in BRCA1/2 and PALB2 genes in 2769 unselected breast cancer patients in China. Int. J. Cancer. 2019, 145, 1517-1528. [CrossRef] [PubMed]

11. Heikkinen, T.; Kärkkäinen, H.; Aaltonen, K.; Milne, R.L.; Heikkilä, P.; Aittomäki, K.; Blomqvist, C.; Nevanlinna, H. The breast cancer susceptibility mutation PALB2 1592delT is associated with an aggressive tumor phenotype. Clin. Cancer Res. 2009, 15, 3214-3322. [CrossRef] [PubMed]

12. Southey, M.C.; Teo, Z.L.; Dowty, J.G.; Odefrey, F.A.; Park, D.J.; Tischkowitz, M.; Sabbaghian, N.; Apicella, C.; Byrnes, G.B.; Winship, I.; et al. A PALB2 mutation associated with high risk of breast cancer. Breast Cancer Res. 2010, 12, R109. [CrossRef]

13. Rahman, N.; Seal, S.; Thompson, D.; Kelly, P.; Renwick, A.; Elliott, A.; Reid, S.; Spanova, K.; Barfoot, R.; Chagtai, T.; et al. PALB2, which encodes a BRCA2 interacting protein, is a breast cancer susceptibility gene. Nat. Genet. 2007, 39, 165-167. [CrossRef] [PubMed]

14. Kwong, A.; Shin, V.Y.; Chen, J.; Cheuk, I.W.Y.; Ho, C.Y.S.; Au, C.H.; Chan, K.; Ngan, H.; Chan, T.L.; Ford, J.M.; et al. Germline Mutation in 1338 BRCA-Negative Chinese Hereditary Breast and/or Ovarian Cancer Patients: Clinical Testing with a Multigene Test Panel. J. Mol. Diagn. 2020, 22, 544-554. [CrossRef] [PubMed]

15. Wu, Y.; Ouyang, T.; Li, J.; Wang, T.; Fan, Z.; Fan, T.; Lin, B.; Xu, Y.; Xie, Y. Spectrum and clinical relevance of PALB2 germline mutations in 7657 Chinese BRCA1/2-negative breast cancer patients. Breast Cancer Res. Treat. 2020, 179, 605-614. [CrossRef] [PubMed]

16. Isaac, D.; Karapetyan, L.; Tamkus, D. Association of germline PALB2 mutation and response to platinum-based chemotherapy in metastatic breast cancer: A case series. JCO Precis. Oncol. 2018, 2, 1-5. [CrossRef]

17. Kuemmel, S.; Harrach, H.; Schmutzler, R.K.; Kostara, A.; Ziegler-Löhr, K.; Dyson, M.H.; Chiari, O.; Reinisch, M. Olaparib for metastatic breast cancer in a patient with a germline PALB2 variant. NPJ Breast Cancer 2020, 6, 31. [CrossRef]

18. Dicks, E.; Song, H.; Ramus, S.J.; Oudenhove, E.V.; Tyrer, J.P.; Intermaggio, M.P.; Kar, S.; Harrington, P.; Bowtell, D.D.; Group, A.S.; et al. Germline whole exome sequencing and large-scale replication identifies FANCM as a likely high grade serous ovarian cancer susceptibility gene. Oncotarget 2017, 8, 50930-50940. [CrossRef]

19. Ng, P.S.; Boonen, R.A.; Wijaya, E.; Chong, C.E.; Sharma, M.; Knaup, S.; Mariapun, S.; Ho, W.K.; Lim, J.; Yoon, S.Y.; et al. Characterisation of protein-truncating and missense variants in PALB2 in 15768 women from Malaysia and Singapore. J. Med. Genet 2021. [CrossRef]

20. NM_024675.3(PALB2):c.444delG (p.Lys149Serfs). Available online: https://www.ncbi.nlm.nih.gov/clinvar/RCV000576472 (accessed on 14 May 2021).

21. NM_024675.3(PALB2):c.839del (p.Asn280fs). Available online: https:/ / www.ncbi.nlm.nih.gov/clinvar/variation/480243/ (accessed on 19 August 2021).

22. NM_024675.3(PALB2):c.1048C>T (p.Gln350Ter). Available online: https://www.ncbi.nlm.nih.gov/clinvar/variation/410191/ (accessed on 14 May 2021).

23. NM_024675.3(PALB2):c.1059del (p.Lys353fs). Available online: https://www.ncbi.nlm.nih.gov/clinvar/variation/182745/ (accessed on 14 May 2021).

24. NM_024675.4(PALB2):c.1240C>T (p.Arg414Ter). Available online: https://www.ncbi.nlm.nih.gov/clinvar/variation/128117/ (accessed on 14 May 2021). 
25. NM_024675.3(PALB2):c.1592del (p.Leu531fs). Available online: https://www.ncbi.nlm.nih.gov/clinvar/variation/126609/ (accessed on 14 May 2021).

26. NM_024675.3(PALB2):c.2108T>G (p.Leu703Ter) AND Familial cancer of breast. Available online: https://www.ncbi.nlm.nih. gov/clinvar/68467629/ (accessed on 14 May 2021).

27. NM_024675.3(PALB2):c.444del (p.Lys149fs) AND Familial cancer of breast. Available online: https://www.ncbi.nlm.nih.gov/ clinvar/68737907/ (accessed on 14 May 2021).

28. NM_024675.3(PALB2):c.2594C>G (p.Ser865Ter). Available online: https://www.ncbi.nlm.nih.gov/clinvar/variation/241547/ (accessed on 14 May 2021).

29. Ng, P.S.; Pan, J.W.; Ahmad Zabidi, M.M.; Rajadurai, P.; Yip, C.H.; Reuda, O.M.; Dunning, A.M.; Antoniou, A.C.; Easton, D.F.; Caldas, C.; et al. Characterisation of PALB2 tumours through whole-exome and whole-transcriptomic analyses. NPJ Breast Cancer 2021, 7, 46. [CrossRef]

30. NM_024675.3(PALB2):c.2968G>T (p.Glu990Ter). Available online: https://www.ncbi.nlm.nih.gov/clinvar/variation/231227/ (accessed on 19 August 2021).

31. NM_024675.3(PALB2):c.3114-1G>A. Available online: https://www.ncbi.nlm.nih.gov/clinvar/variation/265552/ (accessed on 14 May 2021).

32. NM_024675.3(PALB2):c.3256C>T (p.Arg1086Ter). Available online: https:/ / www.ncbi.nlm.nih.gov/clinvar/variation/126729/ (accessed on 14 May 2021).

33. NM_024675.4(PALB2):c.3507_3508del (p.His1170fs). Available online: https://www.ncbi.nlm.nih.gov/clinvar/variation/140978/ (accessed on 19 August 2021).

34. Cybulski, C.; Kluźniak, W.; Huzarski, T.; Wokołorczyk, D.; Kashyap, A.; Jakubowska, A.; Szwiec, M.; Byrski, T.; Dębniak, T.; Górski, B.; et al. Clinical outcomes in women with breast cancer and a PALB2 mutation: A prospective cohort analysis. Lancet Oncol. 2015, 16, 638-644. [CrossRef]

35. Tung, N.; Lin, N.U.; Kidd, J.; Allen, B.A.; Singh, N.; Wenstrup, R.J.; Hartman, A.R.; Winer, E.P.; Garber, J.E. Frequency of germline mutations in 25 cancer susceptibility genes in a sequential series of patients with breast cancer. J. Clin. Oncol. 2016, 34, 1460-1468. [CrossRef]

36. Zhou, J.; Wang, H.; Fu, F.; Li, Z.; Feng, Q.; Wu, W.; Liu, Y.; Wang, C.; Chen, Y. Spectrum of PALB2 germline mutations and characteristics of PALB2-related breast cancer: Screening of 16,501 unselected patients with breast cancer and 5890 controls by next-generation sequencing. Cancer. 2020, 126, 3202-3208. [CrossRef]

37. Castéra, L.; Harter, V.; Muller, E.; Krieger, S.; Goardon, N.; Ricou, A.; Rousselin, A.; Paimparay, G.; Legros, A.; Bruet, O.; et al. Landscape of pathogenic variations in a panel of 34 genes and cancer risk estimation from $5131 \mathrm{HBOC}$ families. Genet. Med. 2018, 20, 1677-1686. [CrossRef]

38. Breast Cancer Association Consortium; Dorling, L.; Carvalho, S.; Allen, J.; González-Neira, A.; Luccarini, C.; Wahlström, C.; Pooley, K.A.; Parsons, M.T.; Fortuno, C.; et al. Breast Cancer Risk Genes-Association Analysis in More than 113,000 Women. N. Engl. J. Med. 2021, 384, 428-439. [CrossRef] [PubMed]

39. Erkko, H.; Dowty, J.G.; Nikkilä, J.; Syrjäkoski, K.; Mannermaa, A.; Pylkäs, K.; Southey, M.C.; Holli, K.; Kallioniemi, A.; Jukkola-Vuorinen, A.; et al. Penetrance analysis of the PALB2 c.1592delT founder mutation. Clin. Cancer Res. 2008, 14, 4667-4671. [CrossRef] [PubMed]

40. Kluska, A.; Balabas, A.; Piatkowska, M.; Czarny, K.; Paczkowska, K.; Nowakowska, D.; Mikula, M.; Ostrowski, J. PALB2 mutations in BRCA1/2-mutation negative breast and ovarian cancer patients from Poland. BMC Med. Genomics. 2017, 10, 14. [CrossRef] [PubMed]

41. Foules, W.D.; Ghadirian, P.; Akbari, M.R.; Hamel, N.; Giroux, S.; Sabbaghian, N.; Darnel, A.; Royer, R.; Poll, A.; Fafard, E.; et al. Identification of a novel truncating PALB2 mutation and analysis of its contribution to early-onset breast cancer in French-Canadian women. Breast Cancer Res. 2007, 9, R83. [CrossRef] [PubMed]

42. Pern, F.; Bogdanova, N.; Schürmann, P.; Lin, M.; Ay, A.; Länger, F.; Hillemanns, P.; Christiansen, H.; Park-Simon, T.W.; Dörk, T. Mutation analysis of BRCA1, BRCA2, PALB2 and BRD7 in a hospital-based series of German patients with triple-negative breast cancer. PLoS ONE 2021, 7, e47993. [CrossRef] [PubMed]

43. Cote, S.; Arcand, S.L.; Royer, R.; Nolet, S.; Mes-Masson, A.M.; Ghadirian, P.; Foulkes, W.D.; Tischkowitz, M.; Narod, S.A.; Provencher, D.; et al. The BRCA2 c.9004G > A (E2002K) variant is likely pathogenic and recurs in breast and/or ovarian cancer families of French Canadian descent. Breast Cancer Res. Treat. 2012, 131, 333-340. [CrossRef]

44. Yoshikiyo, K.; Kratz, K.; Hirota, K.; Nishihara, K.; Takata, M.; Kurumizaka, H.; Horimoto, S.; Takeda, S.; Jiricny, J. KIAA1018/FAN1 nuclease protects cells against genomic instability induced by interstrand cross-linking agents. Proc. Natl. Acad. Sci. USA 2010, 107, 21553-21557. [CrossRef]

45. Gruber, J.J.; Afghani, A.; Hatton, A.; Scott, D.; McMillan, A.; Ford, J.M.; Telli, M.L. Talazoparib beyond BRCA: A phase II trial of talazoparib monotherapy in BRCA1 and BRCA2 wild-type patients with advanced HER2-negative breast cancer or other solid tumors with a mutation in homologous recombination (HR) pathway genes. J. Clin. Oncol. 2019, 37, 3006. [CrossRef]

46. Tung, N.M.; Robson, M.E.; Ventz, S.; Santa-Maria, C.A.; Nanda, R.; Marcom, P.K.; Shah, P.D.; Ballinger, T.J.; Yang, E.S.; Vinayak, S.; et al. TBCRC 048: Phase II Study of Olaparib for Metastatic Breast Cancer and Mutations in Homologous Recombination-Related Genes. J. Clin. Oncol. 2020, 38, 4274-4282. [CrossRef] 
47. Grellety, T.; Peyraud, F.; Sevenet, N.; Tredan, O.; Dohollou, N.; Barouk-Simonet, E.; Kind, M.; Longy, M.; Blay, J.Y.; Italiano, A. Dramatic response to PARP inhibition in a PALB2-mutated breast cancer: Moving beyond BRCA. Ann. Oncol. 2020, 31, 822-823. [CrossRef]

48. USA National Library of Medicine. 2021. Available online: https:/ / clinicaltrials.gov/ct2/show /NCT04756765?term=PALB2 \&cond=breast+cancer\&draw $=2 \&$ rank $=2$ (accessed on 15 January 2021).

49. NCCN Clinical Practice Guidelines in Oncology (NCCN Guidelines). 2021. Genetic/Familial High-Risk Assessment: Breast and Ovarian. Available online: https://www2.tri-kobe.org/nccn/guideline/gynecological/english/genetic_familial.pdf (accessed on 15 January 2021).

50. Tischkowitz, M.; Balmaña, J.; Foulkes, W.D.; James, P.; Ngeow, J.; Schmutzler, R.; Voian, N.; Wick, M.J.; Stewart, D.R.; Pal, T.; et al. ACMG Professional Practice and Guidelines Committee. Management of individuals with germline variants in PALB2: A clinical practice resource of the American College of Medical Genetics and Genomics (ACMG). Genet. Med. 2021, 23, 1416-1423. [CrossRef]

51. Kotsopoulos, J.; Sopik, V.; Rosen, B.; Fan, I.; McLaughlin, J.R.; Risch, H.; Sun, P.; Narod, S.A.; Akbari, M.R. Frequency of germline PALB2 mutations among women with epithelial ovarian cancer. Fam. Cancer. 2017, 16, 29-34. [CrossRef]

52. Janssen, B.; Bellis, S.; Koller, T.; Tischkowitz, M.; Liau, S.S. A systematic review of predicted pathogenic PALB2 variants: An analysis of mutational overlap between epithelial cancers. J. Hum. Genet. 2020, 65, 199-205. [CrossRef] [PubMed]

53. Kwong, A.; Shin, V.Y.; Au, C.H.; Law, F.B.F.; Ho, D.N.; Wong, A.T.C.; Lau, S.S.; To, R.M.; Choy, G.; Ford, J.M.; et al. Detection of germline mutation in hereditary breast and/ovarian cancers by next-generation sequencing on a four gene panel. J. Mol. Diagn. 2016, 18, 580-594. [CrossRef] [PubMed]

54. Neben, C.L.; Zimmer, A.D.; Stedden, W.; van den Akker, J.; O'Connor, R.; Chan, R.C.; Chen, E.; Tan, Z.; Leon, A.; Ji, J.; et al. Multi-Gene Panel Testing of 23,179 Individuals for Hereditary Cancer Risk Identifies Pathogenic Variant Carriers Missed by Current Genetic Testing Guidelines. J. Mol. Diagn. 2019, 21, 646-657. [CrossRef] [PubMed]

55. The 1000 Genomes Project Consortium: A global reference for human genetic variation. Nature 2015, 526, 68-74. [CrossRef] [PubMed] 